and young women as a solitary lesion. These tumours, including those that are metastatic, behave quiescently in the vast majority of patients. This case demonstrates the unusual presentation of metastatic solid pseudopapillary neoplasm of the pancreas masquerading as a primary liver tumour.

\section{AN EXTRAORDINARILY LARGE ABDOMINAL MASS OF UNKNOWN ORIGIN: A CASE REPORT}

Serena J.K. Park, Rachael van der Griend, Matthew Drake Anatomical Pathology Department, Canterbury Health Laboratories, Christchurch, New Zealand

We describe a case of a 45-year-old woman who presented with abdominal pain, vomiting and early satiety. Radiological investigation showed a multiloculated cystic mass with thickened septae filling most of the abdomen and pelvis. CA-125 was also raised, which led to a pre-operative diagnosis of ovarian malignancy.

At laparotomy, there was a large multicystic mass arising in the region of transverse colon mesentery. It extended inferiorly to fill the pelvis and extended superiorly to lie directly on the top of pancreas, which led the surgeons to suspect pancreatic origin for the mass. However, at the completion of the resection, which also required right hemicolectomy, the nature of the mass was still unclear.

Histopathological examination showed variably-sized cystic spaces lined by endothelium and containing proteinaceous fluid, with the cells of the endothelial lining expressing lymphatic markers. The morphological and immunohistochemical features confirmed the diagnosis of cystic mesenteric lymphangioma. However, the differential diagnosis for such a large mass in this location is broad, and ranges from completely benign conditions to aggressive malignancy. Details of the final diagnosis and the differential diagnoses will be discussed.

\section{TESTICULAR PAIN WITH A TWIST - A CASE REPORT}

Elizabeth Paver, Yvonne Bogun

Department of Anatomical Pathology, The Canberra Hospital, ACT, Australia

We present the case of an otherwise well, 19-year-old man admitted with acute, severe left testicular pain. He had no significant past medical history. Haematological investigations (including inflammatory markers) and urine culture were normal, and tumour markers were negative. Testicular ultrasound revealed an abnormal region of hypoechogenicity within the left testis, the differentials for which included neoplasm, haematoma or abscess, and he underwent a radical orchiectomy. Pathological examination revealed a soft, tan, wedge-shaped lesion, identified microscopically as an area of infarction, associated with vasculitis of the medium-sized vessels both within the infarcted area as well as in the distant, unaffected stroma.

Vasculitis of the testis is a rare condition, presenting as acute testicular pain secondary to localised infarction. Clinically it can mimic cancer. While in most patients it occurs as an isolated event that is cured by surgery, up to $25 \%$ of cases may be associated with systemic vasculitis. This case illustrates the difficulty sometimes faced in diagnosing testicular lesions preoperatively, and serves as a reminder that all patients presenting with testicular vasculitis should be clinically investigated for systemic disease.

\section{YOU MAY NOT ALWAYS GET THE GIST...A CASE REPORT}

Elizabeth Paver $^{1}$, Alexandra Currie ${ }^{1,2}$, Sanjiv Jain ${ }^{1,2}$

${ }^{1}$ Department of Anatomical Pathology, ACT Pathology, The

Canberra Hospital, and ${ }^{2}$ Australian National University

Medical School, ACT, Australia

A 39-year-old man presented with syncope and anaemia on a background of episodic haematemesis and melaena. Gastroscopy revealed bleeding from a pre-pyloric, submucosal tumour that was suspicious for a gastrointestinal stromal tumour (GIST). A distal antrectomy was performed. A frozen section at the time of surgery was not supportive of a GIST, and diagnosis was deferred. Paraffin sections with immunohistochemical staining revealed a benign glomus tumour of the stomach.

Glomus tumours (GTs) are mesenchymal neoplasms which usually occur in the subcutaneous tissue of the distal extremities, but which have rarely been described in a variety of sites such as the stomach, soft tissues, nasal cavity, trachea and liver. They are thought to differentiate towards the modified smooth muscle cells of the glomus body, involved in regulating arteriolar blood flow. While they are generally considered benign, rare reports of malignant transformation have been published. Intra-operative diagnosis can be challenging if pathologists are unaware of the existence of this entity in unusual sites. We hope that this case will serve as a reminder that glomus tumours may occur in unusual sites, in particular, the submucosa of the stomach.

\section{A HURTHLE CELL NEOPLASM WITH NOVEL HISTOLOGICAL FEATURES}

Benjamin Popp, Stephen Chan

Dorevitch Pathology, Western Hospital, Footscray, Vic, Australia

This is a case of an 85-year-old woman with a 3 year history of a right thyroid mass which had recently increased in size. CT imaging demonstrated that the lesion had increased from $61 \mathrm{~mm}$ to $100 \mathrm{~mm}$ with retrosternal extension, surrounding lymphadenopathy and potential metastasis to the lung. Serial slicing of the thyroid demonstrated a well circumscribed nodule with a fleshy pink cut surface containing areas of haemorrhage and necrosis. Sections of the lesion showed a trabecular architecture consisting of fine fibrovascular cores lined by large cells containing centrally located large round nuclei with prominent nucleoli, abundant oncocytic basal cytoplasm and a cleared luminal cytoplasm with a microvesicular appearance. Examination of the surrounding capsule showed evidence of invasion. The tumour cells were TTF-1 positive, weakly thyroglobulin positive and focally CK19 positive. Staining for calcitonin and HBME1 was negative. Columnar cell variant of papillary thyroid carcinoma was considered as a differential diagnosis but no features of papillary thyroid carcinoma were present. Hurthle cell neoplasms are known to contain clear cell areas. This case highlights a novel histological variation of a Hurthle cell neoplasm with a biphasic cytoplasm showing both oncocytic and clear cell features. 